\title{
Kiwifruit size influences softening rate during storage
}

\author{
Carlos H. Crisosto a David Garner \ Katia Saez
}

\begin{abstract}
Large ( 101 grams), medium ( 93 grams) and small ( 81 grams) 'Hayward' kiwifruits were stored in either ethylene-free air or in a controlled atmosphere (CA) of 5\% carbon dioxide $\left(\mathrm{CO}_{2}\right)$ and $2 \%$ oxygen $\left(\mathrm{O}_{2}\right)$ at $32^{\circ} \mathrm{F}$ for 16 weeks. Under both storage conditions, large fruit had a slower rate of softening than smaller fruit. Air-stored kiwifruit softened approximately 2.6 times faster than CA-stored fruit. Under air conditions, large, medium and small kiwifruit reached $5.0 \mathrm{lbf}$ (the minimum pounds firmness required for packaging with minimal bruising) by 12,10 and 8 weeks, respectively, while those stored under CA conditions softened to $5.0 \mathrm{lbf}$ by 49, 30 and 20 weeks. Understanding the relationship between fruit size and the rate of softening under air and $C A$ conditions will help cold storage managers safely monitor kiwifruit softening during bin storage.
\end{abstract}

Any technology that reduces the cost of repackaging kiwifruit will increase profit to the growers and packers. Traditionally, kiwifruit are picked, sorted, sized and packed immediately into containers of various sizes. Generally, after 2 to 3 months storage, kiwifruit are repackaged to remove fruit infected with fungus decay (Botrytis cineria), and to satisfy market conditions at the time. It is very difficult for packers to predict the container/size combination that will be required by buyers 2 to 3 months after harvest.

To eliminate repackaging costs, field-run kiwifruit can be stored in bins for up to 3 months before packaging in the final container. Short-term bin storage would reduce packaging pressure, cooling time, ethylene contamination and repackaging costs. However, it has been demonstrated (Mitchell 1990) that kiwifruit are more susceptible to vibration damage during packaging and/or transportation when they soften below $5.0 \mathrm{lbf}$

(pounds firmness). For this reason, we investigated the relationship between fruit size, storage atmosphere and the rate of softening under both air-stored and controlled-atmosphere conditions.

\section{Kiwifruit source}

'Hayward' kiwifruit grown commercially in the Visalia area, were harvested at a late maturity (Nov. 11, 1996) into 18-inch-high plastic bins and immediately sized, graded and stored. Only small kiwifruit $(\leq 81$ grams) were commercially bin-stored in air after a light brushing, sorting and grading. At the same time, kiwifruit samples of different sizes from the same lot were collected directly from the packingline after packaging (brushing, grading, sorting) and immediately transported to the F. Gordon Mitchell Postharvest Laboratory at the Kearney Agricultural Center in Parlier for the controlled-atmosphere (CA) experiment. Fruit quality evaluations included measurements of soluble solids concentration (SSC), acidity, firmness, water loss, decay and bruising. At harvest, kiwifruit had approximately $12.0 \mathrm{lbf}$ (measured with an $8-\mathrm{mm}$ penetrometer tip), $11 \%$ SSC and $1.9 \%$ titratable acidity (TA).

\section{CA laboratory experiment}

Since ethylene concentrations as low as 10 parts per billion induce rapid kiwifruit softening during cold storage, we investigated the rate of fruit softening under ethylene-free CA

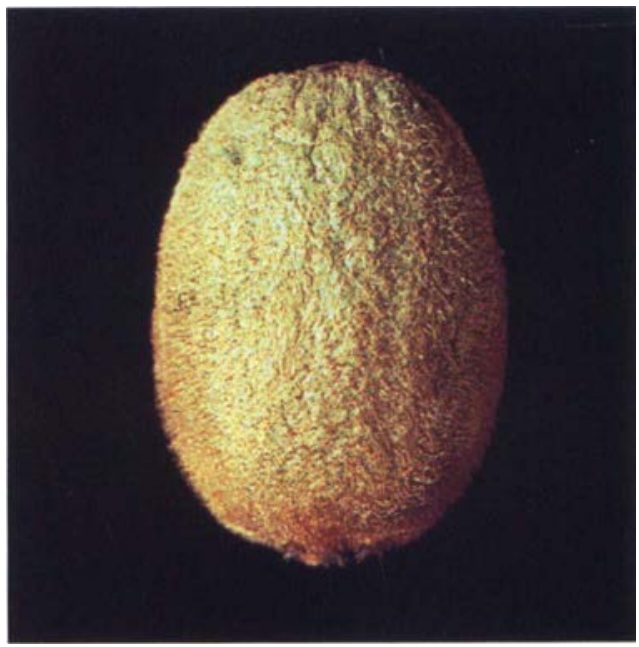

Kiwifruit that has lost approximately $4 \%$ of its original weight begins to shrivel.

and air-storage conditions for small, medium and large fruit under controlled laboratory conditions, with a factorial design arranged in a split plot. Three replications of 10 fruit for each storage/size combination were withdrawn after $0,2,4,6,8,10,12,14$ and 16 weeks in storage. Upon arrival to the laboratory, kiwifruit were aircooled to approximately $35^{\circ} \mathrm{F}$ within 6 hours.

The kiwifruit were then stored in 2.5-gallon jars under a continuous flow of either ethylene-free air or $5 \%$ $\mathrm{CO}_{2}$ and $2 \% \mathrm{O}_{2}$ at $32^{\circ} \mathrm{F}$. We established flow rates and gas mixtures using a mixing board with micrometering valves. Supply and exhaust gas composition were monitored using a gas chromatograph equipped with a thermal conductivity detector for $\mathrm{O}_{2}$ and $\mathrm{CO}_{2}$, and a gas chromatograph equipped with a flame ionization detector for ethylene $\left(\mathrm{C}_{2} \mathrm{H}_{4}\right)$.

At each sampling date, fruit were warmed to ambient temperature $\left(68^{\circ} \mathrm{F}\right)$ for evaluation of firmness and SSC. We removed the skin from opposite cheeks of each fruit and measured the firmness using a UC firmness tester with an 8-mm tip. Then, a longitudinal wedge (from stem end to calyx end) was removed from each fruit, pressed through cheesecloth, and the SSC of the juice measured with a temperaturecompensated refractometer.

The data were subjected to analysis of variance (ANOVA), mean separation and regression analysis where appropriate using the statistical analysis software (SAS) program. 


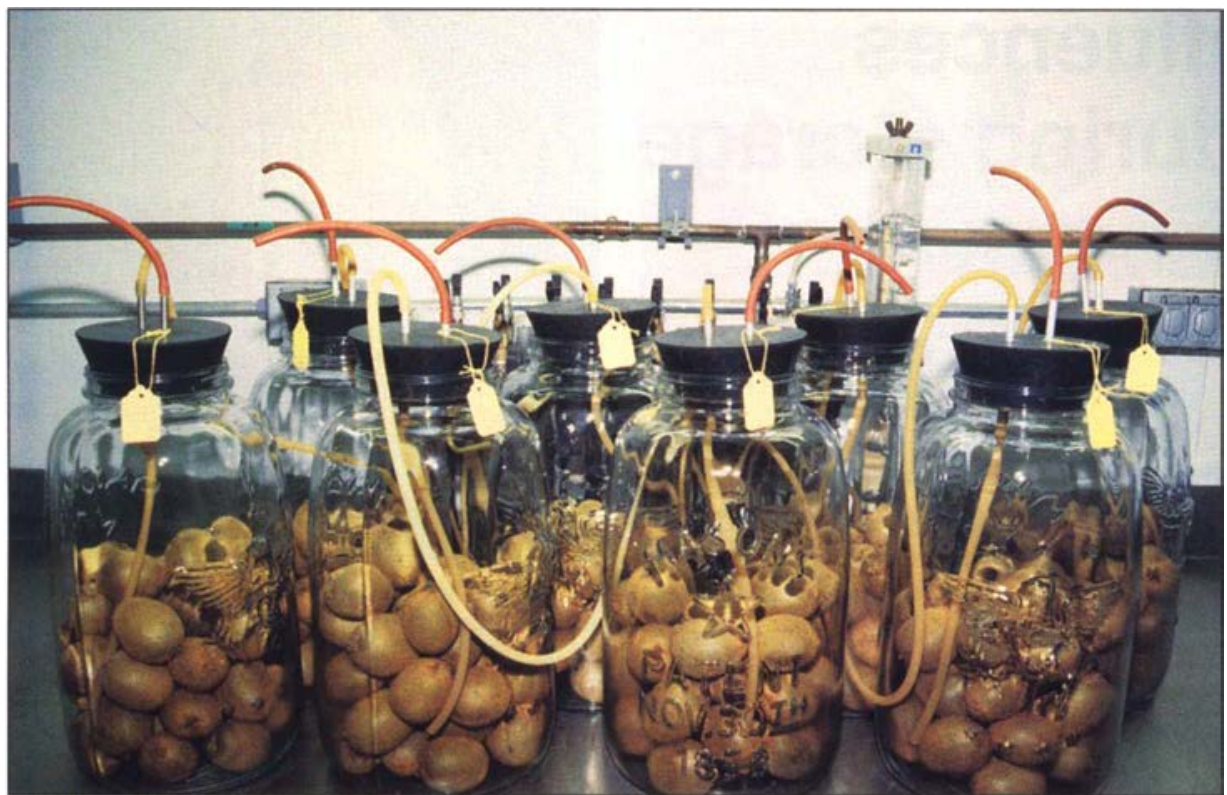

In lab tests, air-stored kiwifruit softened approximately 2.6 times faster than controlled atmosphere-stored kiwifruit.

\section{Bin air-storage trial}

We used a total of 45 bins, with small (size 45) kiwifruit stored in ethylene-free air at $32^{\circ}$ to $33^{\circ} \mathrm{F}$ for 10 weeks before packaging. Fifteen bins (replications) of kiwifruit were randomly packed after 4, 7 and 10 weeks of storage. Bins were stacked in storage up to nine high. The top bins were

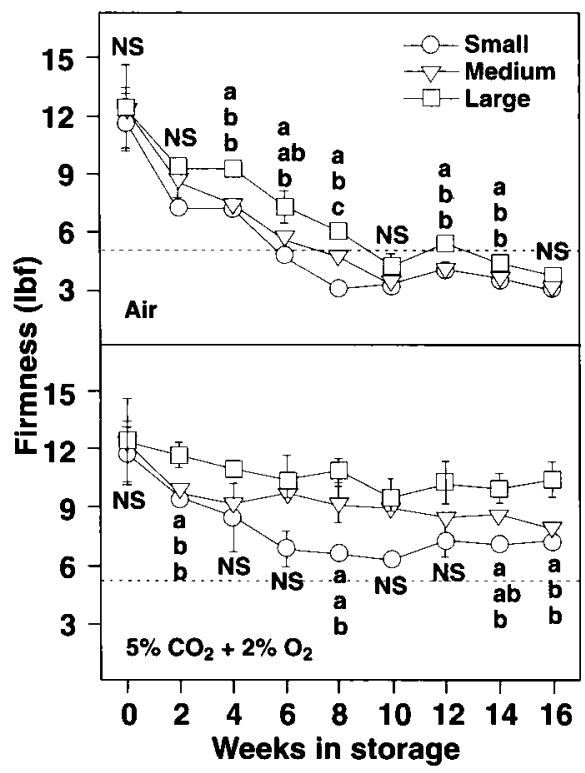

Fig. 1. Cheek firmness of small (size 45,

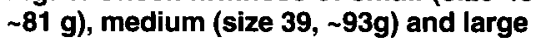
(size 36, $101 \mathrm{~g}$ ) kiwifruit stored in ethylenefree air or $5 \% \mathrm{CO}_{2}+2 \% \mathrm{O}_{2}$. Different letters indicate a significant difference in firmness at that date $(L S D=0.05)$. covered by a tarp to minimize water loss. Prior to dumping on the packing line, kiwifruit samples were collected for water loss evaluations from wire baskets previously prepared and placed in the tops of the bins. Then the 15 bins per treatment were dumped and packed in a commercial operation. At each packaging date, 30 fruit per each of the 15 replications were

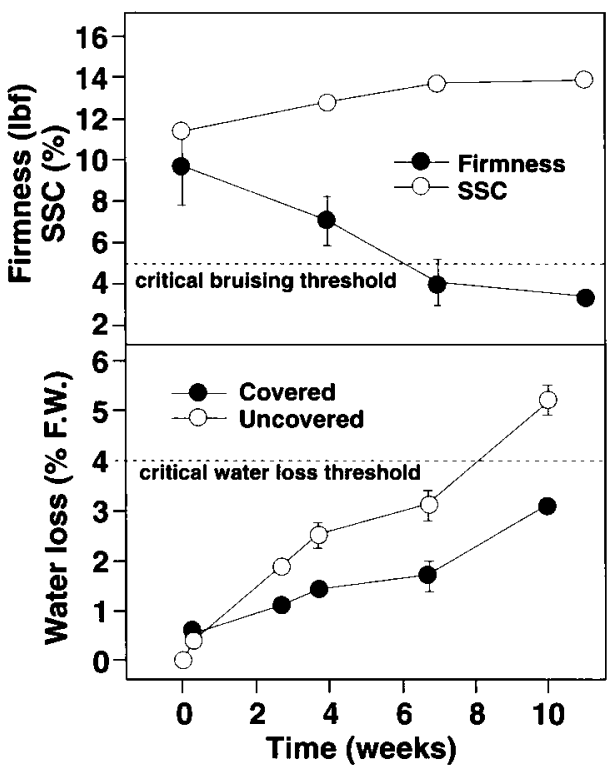

Fig. 2. Top, changes in firmness and soluble solids concentrations of binstored kiwifruit. Bottom, weight loss of covered and uncovered kiwifruit in the tops of bins. Vertical bars represent standard deviation. sampled from the bottom of the bin after dumping. Kiwifruit samples were immediately taken to the laboratory for firmness, SSC, bruising and decay evaluation.

\section{Interaction between size, storage}

\section{Controlled atmosphere experi-}

ment. Because there was a significant interaction $(P$-value $<0.001)$ between storage atmosphere and kiwifruit size for changes in firmness during the 16-week storage period, firmness measurements are presented for individual treatments (storage/size). Kiwifruit softening during the 16-week cold storage period was related to fruit size and storage atmosphere conditions (fig. 1). The increase in SSC during storage, however, was independent of the fruit size and the storage atmosphere conditions (data not shown). Regression equations significantly described firmness changes during storage (weeks) for each of the storage condition/size combinations (table 1).

Under both storage conditions, large fruit had a slower rate of softening than smaller kiwifruit. The rate of fruit softening described by the slope of the regression equations was always slower under CA than air conditions (table 1). Air-stored kiwifruit softened approximately 2.6 times faster than CA-stored fruit. After 16 weeks of CA storage, large, medium and small kiwifruit had firmnesses of 12.0, 9.0 and $9.0 \mathrm{lbf}$, respectively. Large, medium and small air-stored fruit all had firmnesses of approximately $3.0 \mathrm{lbf}$ after this same period of time (fig. 1).

Because kiwifruit are more susceptible to vibration damage during packaging and/or transportation when they soften below $5.0 \mathrm{lbf}$, we ran our regression equations to predict the number of weeks to reach this firmness. Under air-storage conditions, large, medium and small kiwifruit from this orchard reached $5.0 \mathrm{lbf}$ by 12,10 and 8 weeks, respectively. In contrast, large, medium and small kiwifruit under CA storage conditions were estimated to reach $5.0 \mathrm{lbf}$ by 49 , 30 and 20 weeks, respectively. Thus, 
the length of time the fruit could be held safely in cold storage prior to packaging was related to fruit size and storage atmosphere.

Bin cold storage. During the storage period, ethylene levels were always lower than 10 parts per billion (ppb). For small fruit sampled from the bottoms of the bins, fruit firmness decreased from approximately $12.0 \mathrm{lbf}$ to $4.0 \mathrm{lbf}$ after 10 weeks cold storage (fig. 2). The greatest drop in fruit firmness occurred within the first 4 weeks of storage.

Using the regression equation ( $P$ value $\left.<0.0001, r^{2}=0.75\right)$ for the relationship between changes in firmness versus weeks in air storage for the bins, we predicted that after 7 weeks our kiwifruit reached the critical firmness level for packaging $(5.0 \mathrm{lbf})$. The kiwifruit lost approximately $0.6 \mathrm{lbf}$ per week during bin storage in air at $32^{\circ} \mathrm{F}$. The difference in time to reach $5.0 \mathrm{lbf}$ can be explained by the different storage conditions in the commercial situation. Thus, this regression equation can be used as a tool to predict the potential storage time under optimum conditions (ethylene-free air at $32^{\circ} \mathrm{F}$ ). Firmness measurements should be done during the storage period to determine the end of the bin storage period and a safe packaging date.

Exposed kiwifruit in the tops of the bins (the worst-case scenario for fruit damage) lost weight at a constant rate during the cold-storage period (fig. 2). The use of canvas covers over the tops of the bins reduced the rate of water loss by nearly half. In previous work, we have observed kiwifruit shriveling only after fruit water-loss values exceeded $4.0 \%$ of the initial fruit fresh weight. Bruising incidence after packaging varied from $1.0 \%$ to $4.1 \%$. More bruising occurred on the last two packing dates when kiwifruit had firmness values equal to or less than 4.0 lbf. Decay was not observed during this cold-storage trial.

We observed a difference in the rates of fruit softening under laboratory versus bin air storage. Under laboratory conditions, it took 1 week longer to reach $5.0 \mathrm{lbf}$ than under commercial bin storage conditions. This may be due to better circulation of air around the fruit under laboratory conditions, or possibly the difference in the weight of fruit upon fruit. It is important to point out that the softening rate of a given size of kiwifruit during ethylene-free storage is also influenced by orchard factors. For instance, Johnson and others (1997) have reported the influence of different nitrogen application rates and the nitrogen/calcium ratios on kiwifruit softening.

\section{Softening-rate factors}

The rate of kiwifruit softening is related to kiwifruit size, storage atmosphere conditions and orchard factors. In general, large kiwifruit had a slower rate of softening than smaller kiwifruit. Thus, large kiwifruit have longer bin storage potential than medium and small kiwifruit.

However, this bin storage period may vary according to orchard influ-

TABLE 1. Regression equations showing the relationships between firmness and storage period (weeks) for three sizes $(45,39,36)$ of kiwifruit stored under air or controlled atmosphere conditions

\begin{tabular}{lcccc}
\hline \hline Parameter/treatment & Intercept & Slope & $r^{2}$ & P-value \\
\hline Firmness & & & & \\
Air 45 & 8.7 & -0.44 & 0.68 & 0.0001 \\
Air 39 & 9.8 & -0.50 & 0.79 & 0.0001 \\
Air 36 & 10.7 & -0.49 & 0.31 & 0.0001 \\
Controlled atmosphere 45 & 9.4 & -0.22 & 0.53 & 0.0024 \\
Controlled atmosphere 39 & 10.7 & -0.19 & 0.17 & 0.0001 \\
Controlled atmosphere 36 & 11.4 & -0.13 & & 0.031 \\
\hline
\end{tabular}

ences. Ethylene-free, cold air storage is a promising technique when there is a short delay (less than 8 weeks) in packaging. $\mathrm{CA}$ cold storage $\left(5 \% \mathrm{CO}_{2}\right.$ and $\left.2 \% \mathrm{O}_{2}\right)$ is a possibility for longterm (more than 8 weeks) storage of kiwifruit prior to packaging. Both technologies are commercially feasible. In both cases, we recommend monitoring firmness changes during storage to determine a safe packaging time. A minimum of $5.0 \mathrm{lbf}$ is suggested as a critical level to prevent bruising during packaging and domestic shipment.

C.H. Crisosto is Extension Postharvest Physiologist, and D. Garner is Staff Research Associate, Pomology Department, UC Davis, located at Kearney Agricultural Center, Parlier; and K. Saez is Assistant Professor with the Statistics and Mathematics Department, University of Concepcion, Concepcion, Chile.

\section{References}

Johnson RS, Mitchell FG, Crisosto $\mathrm{CH}$, et al. 1997. Nitrogen influences kiwifruit storage life. Acta Hort 444(1):285-9.

Mitchell FG. 1990. Postharvest physiology and technology of kiwifruit. Acta Hort 282:291-307. 